TRANSACTIONS OF THE

AMERICAN MATHEMATICAL SOCIETY

Volume 351, Number 6, Pages 2479-2497

$\mathrm{S}$ 0002-9947(99)02495-2

Article electronically published on February 24, 1999

\title{
EMBEDDED SINGULAR CONTINUOUS SPECTRUM FOR ONE-DIMENSIONAL SCHRÖDINGER OPERATORS
}

\author{
CHRISTIAN REMLING
}

\begin{abstract}
We investigate one-dimensional Schrödinger operators with sparse potentials (i.e. the potential consists of a sequence of bumps with rapidly growing barrier separations). These examples illuminate various phenomena related to embedded singular continuous spectrum.
\end{abstract}

\section{INTRODUCTION}

In this paper, we will study one-dimensional Schrödinger equations on the halfline

$$
-y^{\prime \prime}(x)+V(x) y(x)=E y(x), \quad x \in[0, \infty) .
$$

We are interested in the spectral properties of the corresponding operators $H_{\alpha}=$ $-\frac{d^{2}}{d x^{2}}+V(x)$ on $L_{2}([0, \infty))$ with boundary conditions $y(0) \cos \alpha+y^{\prime}(0) \sin \alpha=$ $0, \alpha \in[0, \pi)$ (see e.g. [25] for the basic theory). (1) describes the motion of a quantum mechanical particle, and important physical properties of this system depend directly on the spectral characteristics of the operators $H_{\alpha}$ (for more background information, consult e.g. [14]).

Here, we will analyze two classes of potentials $V$ related to the question of the occurrence of singular continuous spectrum which is embedded in the absolutely continuous spectrum. We will construct potentials so that $\sigma_{a c}\left(H_{\alpha}\right)=[0, \infty)$ and, for a set of boundary conditions $\alpha$ of positive measure, we have that $\sigma_{s c}\left(H_{\alpha}\right) \cap$ $(0, \infty) \neq \emptyset$ (see Theorem 3.3 below). There is also a "complementary" construction: Using similar ideas, we will obtain potentials with $\sigma_{s c}\left(H_{\alpha}\right)=[0, \infty), \sigma_{a c}\left(H_{\alpha}\right) \cap$ $(0, \infty) \neq \emptyset$ for all $\alpha$ (= Theorem 3.5). I do not know of any previous examples for these types of spectral behavior.

It is more difficult to obtain embedded singular continuous spectrum than, say, embedded point spectrum because singular continuous spectrum is related to the subordinacy of the generalized eigenfunction (the notion of subordinacy was introduced and analyzed in [4]; for subsequent developments, see [6], [15]). Here, the term "generalized eigenfunction" simply refers to a solution of (1) which satisfies the boundary condition at $x=0$. More specifically, one encounters the following problem: The singular part of the spectral measure is supported on the set where the generalized eigenfunction is subordinate [4], but even if this set is large, it does not automatically follow that there actually is singular continuous spectrum. Of

Received by the editors May 20, 1997.

1991 Mathematics Subject Classification. Primary 34L40, 81Q10.

Key words and phrases. Schrödinger equation, singular continuous spectrum, subordinate solutions.

(C)1999 American Mathematical Society 
course, as for the point spectrum, the situation is completely different: If the generalized eigenfunction is square integrable, then, trivially, the corresponding energy is an eigenvalue. In fact, potentials with embedded point spectrum which is dense in $[0, \infty)$ have been known for a relatively long time [12], [21].

We will illuminate these remarks with our second class of potentials. These examples have purely absolutely continuous spectrum on $(0, \infty)$, but, as we will prove, the set of energies for which the generalized eigenfunction is subordinate has (local) Hausdorff dimension 1 (= Theorem 4.2b)! (Recall that, by general principles, this set always has Lebesgue measure zero.) Again, this is, to the best of my knowledge, the first explicit example for these phenomena.

All potentials in this paper will be sparse potentials (i.e. mainly $V=0$ ). This is no coincidence: Sparse potentials lead to non-trivial asymptotics of the solutions of (1), and there are powerful methods which allow a detailed analysis of these asymptotics. Here, we will rely mainly on further extensions of the techniques recently developed in [8], [16]. However, sparse potentials were already studied in the celebrated work [13]; further papers using sparse potentials in one way or another are [5], [7], [17], [20], [22]. Although the methods of [8] and [16] are very similar in spirit, the actual implementation of the basic strategy differs in some respects (actually, the results of [8] are stronger). It turns out that both viewpoints are needed here. The examples with embedded singular continuous spectrum make heavy use of the ideas of [8], whereas the second class of examples will be analyzed with an extension of the techniques developed in [16].

This paper is organized as follows: In the next section, we fix the notations and explain the basic strategy for an effective analysis of Schrödinger equations with sparse potentials (as developed in [8], [16]). Sections 3 and 4 investigate in detail the examples described above.

While I was proofreading this paper, I obtained an interesting preprint by Molchanov [11] that also discusses sparse potentials. Molchanov's work has some overlap with [8], [16] and also with Section 3 of this paper.

I would like to thank U. Keich and T. Wolff for useful discussions, the Deutsche Forschungsgemeinschaft for financial support, and I am grateful for the hospitality of Caltech where this work was done.

\section{Preliminaries}

The potentials we will study will have the form

$$
V(x)=\sum_{n=1}^{\infty} g_{n} V_{n}\left(x-a_{n}\right)
$$

with $g_{n}>0, V_{n} \in L_{1}\left(\left[-B_{n}, B_{n}\right]\right)$; the intervals $\left[a_{n}-B_{n}, a_{n}+B_{n}\right]$ are assumed to be disjoint. Let $L_{n}=a_{n}-B_{n}-a_{n-1}-B_{n-1}\left(\right.$ with $\left.a_{0}=B_{0}:=0\right)$ and

$$
I_{n}=\int_{-B_{n}}^{B_{n}}\left|V_{n}(x)\right| d x .
$$

The $a_{n}$ 's can of course be recovered from the $L_{n}, B_{n}$, so it suffices to specify these latter parameters.

Fix $\alpha \in[0, \pi)$, and, for $k>0$, let $y(x, k)$ be the solution of (1) with $E=k^{2}$ and $y(0, k)=-\sin \alpha, y^{\prime}(0, k)=\cos \alpha$. Note that $y$ satisfies the boundary condition $\alpha$ 
at $x=0$. The (modified) Prüfer variables $R(x, k), \varphi(x, k)$ are defined by

$$
\left(\begin{array}{c}
y \\
y^{\prime}
\end{array}\right)=R\left(\begin{array}{c}
\sin \varphi \\
k \cos \varphi
\end{array}\right) \text {. }
$$

Here, we demand that $R>0$ and $\varphi$ be continuous in $x$. Clearly, $R, \varphi$ also depend on $\alpha$, but this dependence will not be made explicit in the notation. We write $R_{n}(k)=R\left(a_{n}-B_{n}, k\right), \varphi_{n}(k)=\varphi\left(a_{n}-B_{n}, k\right)$ (so $R_{n}, \varphi_{n}$ are the Prüfer variables immediately before the $n$th barrier).

$R, \varphi$ obey the equations

$$
\begin{aligned}
(\ln R)^{\prime} & =\frac{V}{2 k} \sin 2 \varphi, \\
\varphi^{\prime} & =k-\frac{V}{k} \sin ^{2} \varphi .
\end{aligned}
$$

In particular, we have $R(x) \equiv R_{n}$ on $x \in\left[a_{n-1}+B_{n-1}, a_{n}-B_{n}\right]$ and

$$
\varphi_{n}(k)=\varphi\left(a_{n-1}+B_{n-1}, k\right)+k L_{n} .
$$

Fix a compact interval $J=\left[k_{1}, k_{2}\right] \subset(0, \infty)$. Then, for $k \in J$, we can integrate (4), (5) over the interval $\left[a_{n}-B_{n}, a_{n}+B_{n}\right]$ and use an elementary Taylor expansion in the parameter $g_{n} I_{n}$. This routine calculation yields (compare [8])

$$
\begin{aligned}
\ln \frac{R_{n+1}(k)}{R_{n}(k)}= & \frac{g_{n}}{2 k} \int_{-B_{n}}^{B_{n}} d x V_{n}(x) \sin 2 \theta_{n}(x, k) \\
& -\frac{g_{n}^{2}}{k^{2}} \int_{-B_{n}}^{B_{n}} d x V_{n}(x) \cos 2 \theta_{n}(x, k) \int_{-B_{n}}^{x} d t V_{n}(t) \sin ^{2} \theta_{n}(t, k) \\
& +O\left(g_{n}^{3} I_{n}^{3}\right),
\end{aligned}
$$

where $\theta_{n}(x, k):=k\left(x+B_{n}\right)+\varphi_{n}(k)$. The remainder $O\left(g_{n}^{3} I_{n}^{3}\right)$ is bounded by $C(J) g_{n}^{3} I_{n}^{3}$ where $C$ is independent of $k \in J$ and $n \in \mathbb{N}$. Finally, let

$$
C_{n}=\max _{k \in J}\left|\frac{d \varphi\left(a_{n-1}+B_{n-1}, k\right)}{d k}\right| .
$$

The crucial observation is that, because of (6), for appropriate probability measures on $J$, the Prüfer angles $\varphi_{n}(k)$ (evaluated modulo $\pi$ ) are approximately independent random variables, provided that $L_{n} \gg C_{n}$. This property can be exploited by computing moments [8] or by investigating the joint distribution of the $\varphi_{n}$ 's [16]. With either method, one can analyze (7) rather accurately.

In order to get quantitative conditions on the $L_{n}$ 's, we need a priori control on the $C_{n}$ 's.

Lemma 2.1 ([8]). Assume $g_{n} I_{n} \rightarrow 0$ and $\left(L_{n}+B_{n}\right) / L_{n+1} \rightarrow 0$. Then there is a constant $C=C(J)$ such that $C_{n} \leq C L_{n-1}$ and

$$
\max _{k \in J}\left|\frac{d^{2} \varphi\left(a_{n-1}+B_{n-1}, k\right)}{d k^{2}}\right| \leq C\left(1+\sum_{m=1}^{n-1} g_{m} I_{m} L_{m}^{2}\right) .
$$

Proof. This follows from a Gronwallization of the differential equations satisfied by $\partial \varphi / \partial k, \partial^{2} \varphi / \partial k^{2}$; see [8, Proposition 5.1] for details. 


\section{EMBEDDED SINGULAR CONTINUOUS SPECTRUM}

Suppose $g_{n} I_{n} \in l_{3}$. Then (7) can be written as

$$
\begin{aligned}
\ln R_{N+1}(k)= & \frac{1}{2 k} \operatorname{Im} \sum_{n=1}^{N} X_{n}(k)-\frac{1}{2 k^{2}} \operatorname{Re} \sum_{n=1}^{N} Y_{n}(k) \\
& +\frac{1}{8 k^{2}} \operatorname{Re} \sum_{n=1}^{N} Z_{n}(k)+\frac{1}{8 k^{2}} \sum_{n=1}^{N} g_{n}^{2}\left|\widehat{V}_{n}(2 k)\right|^{2}+\rho_{N}(k)
\end{aligned}
$$

where $\left|\rho_{N}(k)\right| \leq C$ for all $N \in \mathbb{N}, k \in J$. Here, we have set

$$
\widehat{V}_{n}(2 k)=\int_{-B_{n}}^{B_{n}} V_{n}(x) e^{2 i k x} d x
$$

and

$$
\begin{aligned}
X_{n}(k) & =g_{n} \widehat{V}_{n}(2 k) e^{2 i\left(\varphi_{n}(k)+k B_{n}\right)} \\
Y_{n}(k) & =g_{n}^{2} e^{2 i\left(\varphi_{n}(k)+k B_{n}\right)} \int_{-B_{n}}^{B_{n}} d x V_{n}(x) e^{2 i k x} \int_{-B_{n}}^{x} d t V_{n}(t), \\
Z_{n}(k) & =g_{n}^{2}\left(\widehat{V}_{n}(2 k)\right)^{2} e^{4 i\left(\varphi_{n}(k)+k B_{n}\right)}
\end{aligned}
$$

Since $X_{n}, Y_{n}, Z_{n}$ contain the highly oscillatory factors $\exp \left(2 i \varphi_{n}\right)$, we expect that the leading term of (8) will be $\sum g_{n}^{2}\left|\widehat{V}_{n}(2 k)\right|^{2}$. Thus, by choosing the shape of the bumps $V_{n}$ carefully, we can get non-trivial $k$-dependence of the asymptotics of $R_{N}(k)(N \rightarrow \infty)$. This idea was already used in [8] to construct potentials with $\sigma_{a c}=\left[E_{1}, E_{2}\right] \subset(0, \infty), \sigma_{s c}=[0, \infty) \backslash\left(E_{1}, E_{2}\right)$ (see [8, Theorem 6.3]). As we will see, it is more difficult to obtain embedded singular continuous spectrum.

The barriers $V_{n}$ will have the form

$$
V_{n}(x)=\chi_{\left(-B_{n}, B_{n}\right)}(x) W(x)
$$

where $W$ is the Fourier transform of the characteristic function of a Cantor type set $F$. So, construct $F$ as follows: Let $\delta_{n}>0$ be sufficiently small prescribed numbers. Fix $F_{0}=[a, b] \subset(0, \infty)$ and let $F_{1}=F_{0} \backslash\left(c_{1}^{(0)}-\delta_{0}, c_{1}^{(0)}+\delta_{0}\right)$ where $c_{1}^{(0)}=(a+b) / 2$ is the center of $F_{0}$. In general, if $F_{n}$ is a disjoint union of $2^{n}$ closed intervals with centers $c_{m}^{(n)}\left(m=1, \ldots, 2^{n}\right)$, set $F_{n+1}=F_{n} \backslash \bigcup_{m=1}^{2^{n}}\left(c_{m}^{(n)}-\delta_{n}, c_{m}^{(n)}+\delta_{n}\right)$. The set $F=$ $\bigcap F_{n}$ is closed, nowhere dense and has Lebesgue measure $|F|=b-a-\sum_{n=0}^{\infty} 2^{n+1} \delta_{n}$. We assume that $|F|>0$, and we define

$$
W(x)=\int_{F} \cos 2 k x d k .
$$

Lemma 3.1. Let $F, W(x)$ be as above. Suppose $\sup _{n \in \mathbb{N}} \delta_{n} 2^{\gamma n}<\infty$ for some $\gamma>1$. Then $W(x)=O\left((1+|x|)^{-1+1 / \gamma}\right)$. 
Proof. Let $f_{n}(x)=\int_{F_{n}} \cos 2 k x d k$. Obviously, $\left|f_{n}(x)-f(x)\right| \leq\left|F_{n} \backslash F\right| \rightarrow 0$. Furthermore, by construction of the $F_{n}$,

$$
\begin{aligned}
f_{n+1}(x)-f_{n}(x) & =-\int_{F_{n} \backslash F_{n+1}} \cos 2 k x d k=-\sum_{m=1}^{2^{n}} \int_{c_{m}^{(n)}-\delta_{n}}^{c_{m}^{(n)}+\delta_{n}} \cos 2 k x d k \\
& =-\frac{1}{2 x} \sum_{m=1}^{2^{n}}\left(\sin 2 x\left(c_{m}^{(n)}+\delta_{n}\right)-\sin 2 x\left(c_{m}^{(n)}-\delta_{n}\right)\right) \\
& =-\frac{1}{x} \sin 2 \delta_{n} x \sum_{m=1}^{2^{n}} \cos 2 c_{m}^{(n)} x .
\end{aligned}
$$

In particular, the infinite sum $\sum\left(f_{n+1}(x)-f_{n}(x)\right)$ is absolutely convergent, and thus

$$
\begin{aligned}
|f(x)| & =\left|f_{0}(x)+\sum_{n=0}^{\infty}\left(f_{n+1}(x)-f_{n}(x)\right)\right| \\
& \leq \frac{1}{|x|}\left(1+\sum_{n=0}^{\infty} 2^{n}\left|\sin 2 \delta_{n} x\right|\right) .
\end{aligned}
$$

For $|x|>2^{-\gamma}$, define $N(x) \in \mathbb{N}_{0}$ by demanding $2^{-\gamma}<2^{-\gamma N(x)}|x| \leq 1$. Now the assertion is obtained by considering separately the sums $\sum_{n<N(x)}$ and $\sum_{n \geq N(x)}$ in (12). In the first sum, we use $\left|\sin 2 \delta_{n} x\right| \leq 1$; in the second sum, we estimate $\left|\sin 2 \delta_{n} x\right| \leq 2 \delta_{n}|x|$, and we use that, by hypothesis, $\delta_{n} \leq C 2^{-\gamma n}$. The details of these straightforward computations are left to the reader.

In order to control the first three sums of (8), we will need the following result obtained with the methods discussed in [24]:

Proposition 3.2. Let $X_{n}(n \in \mathbb{N})$ be (complex-valued) random variables. Suppose that there are numbers $\rho_{n} \geq 0$ such that $\sum \rho_{n} \ln ^{2} n<\infty$ and

$$
E\left|X_{n}\right|^{2}+\sum_{m=1}^{n-1}\left|E X_{m} \bar{X}_{n}\right| \leq \rho_{n}
$$

Then $\lim _{N \rightarrow \infty} \sum_{n=1}^{N} X_{n}$ exists almost surely.

Sketch of the proof. This result follows essentially from [24, Theorem 2.4.2]. Of course, we need a version for complex-valued $X_{n}$ 's here, but this extension presents no difficulties. We then obtain the proposition by taking

$$
g(j, N)=2 \sum_{n=j+1}^{j+N} \rho_{n}, \quad h(j, N)=2 \sum_{n=j+1}^{j+N} \rho_{n} \ln ^{2} n
$$

in $[24$, Theorem 2.4.2] and noting that

$$
E\left|\sum_{n=j+1}^{j+N} X_{n}\right|^{2} \leq \sum_{n=j+1}^{j+N} E\left|X_{n}\right|^{2}+2 \sum_{n=j+1}^{j+N} \sum_{m=1}^{n-1}\left|E X_{m} \bar{X}_{n}\right| .
$$

We are now ready to formulate and prove our first main result: 
Theorem 3.3. Let $F$ be a Cantor type set satisfying the assumptions of Lemma 3.1 with $\gamma>6$. Let $g_{n}=n^{-1 / 2}, B_{n}=n^{\beta}$ with $(2-4 / \gamma)^{-1}<\beta<\gamma / 8$, and assume that $n^{\beta /(2 \gamma)} L_{n-1} / L_{n} \rightarrow 0$. Then the half-line Schrödinger operators $H_{\alpha}$ with potential $V$ given by (2), (10), (11) satisfy $\sigma_{a c}\left(H_{\alpha}\right)=\sigma_{\text {ess }}\left(H_{\alpha}\right)=[0, \infty)$, $\sigma_{p}\left(H_{\alpha}\right) \cap(0, \infty)=\emptyset$ and, for a set of boundary conditions $\alpha$ of positive measure, $\sigma_{s c}\left(H_{\alpha}\right) \cap(0, \infty) \neq \emptyset$.

Remarks. 1. The following proof also works under more general assumptions on $\gamma, g_{n}, B_{n}, L_{n}$. However, these general conditions are very clumsy, and it seems pointless to make them explicit.

2. The proof will show that, more precisely, $\sigma_{s c}\left(H_{\alpha}\right) \cap \mathbb{R} \backslash F^{2}=\emptyset$ for almost all $\alpha$, and $\sigma_{s c}\left(H_{\alpha}\right) \cap F^{2} \neq \emptyset$ for a set of $\alpha$ 's of positive measure. Here, $F^{2}=\left\{k^{2}: k \in F\right\}$. Note that the method of [8] (which would establish that the spectrum is purely absolutely continuous on $(0, \infty) \backslash F^{2}$ for every boundary condition) does not work here because of the slow decay of $W(x)$.

Proof. The assertion on $\sigma_{\text {ess }}$ follows from the fact that $V(x) \rightarrow 0$. Furthermore, a standard Gronwall estimate shows that because of the rapid growth of the barrier separations $L_{n},(1)$ has no $L_{2}$-solutions if $E>0$.

Moving on to the non-trivial parts of the proof, we fix $f \in C_{0}^{\infty}(0, \infty)$ with $f \geq 0$, supp $f \cap F=\emptyset$ and $\int f(k) d k=1$. In the first part of the proof, we will show with the aid of Proposition 3.2 that almost surely with respect to the probability measure $d P(k)=f(k) d k$, the right-hand side of (8) remains bounded as $N$ goes to infinity. Using (again) a Gronwall estimate, one can easily extend this statement to $x \in \bigcup_{n}\left[a_{n}-B_{n}, a_{n}+B_{n}\right]$, i.e., $R(x, k)$ is bounded on all of $x \in[0, \infty)$. Running through this argument for two different initial angles $\varphi(0, k)$ then shows that all solutions are bounded for almost every $k \in \operatorname{supp} f$. Since $f$ is arbitrary and $F$ is nowhere dense, this will establish the claim on $\sigma_{a c}$ (by [23, Theorem 5]).

Let

$$
\epsilon_{n}=\left(\int_{|x|>B_{n}}|W(x)|^{2} d x\right)^{1 / 2}
$$

We will need

Lemma 3.4. a) Let $f(k)$ be a bounded function. Then there is a constant $C=$ $C(f)$, such that

$$
\int\left|\widehat{V}_{n}(2 k)-\pi \chi_{F \cup-F}(k) / 2\right|^{2} f(k) d k \leq C \epsilon_{n}^{2} .
$$

b) $\lim _{n \rightarrow \infty} \widehat{V}_{n}(2 k)=\pi \chi_{F \cup-F}(k) / 2$ for almost every $k$.

Proof. a) A calculation using (9), (10), (11), and the Plancherel formula [19, Theorem 9.13(b)] yields

$$
\int\left|\widehat{V}_{n}(2 k)-\pi \chi_{F \cup-F}(k) / 2\right|^{2} d k=\pi \epsilon_{n}^{2} .
$$

This obviously implies the assertion.

b) Since $\gamma>6>2$, Lemma 3.1 shows that $W \in L_{p}$ for some $p<2$. Thus the assertion follows from Zygmund's Theorem on the pointwise convergence of Fourier transforms [26] and the fact that $B_{n} \rightarrow \infty$. 
We are now ready to compute the moments of $X_{n}, Y_{n}, Z_{n}$. Note that Lemma 3.1 implies that $\left(I_{n}\right.$ was defined in eq. $\left.(3)\right)$

$$
I_{n} \leq C n^{\beta / \gamma}, \quad \epsilon_{n} \leq C n^{\beta / \gamma-\beta / 2} .
$$

In particular, we have that $\sum\left(g_{n} I_{n}\right)^{3}<\infty$; thus we may indeed use (8).

Let us first verify that $X_{n}$ satisfies the assumptions of Proposition 3.2. Since we are interested in asymptotic properties (namely, the boundedness of $\sum X_{n}$ almost everywhere), we may restrict $n$ to large values $n \geq n_{0}$. In the sequel, we will do so (if necessary) without explicit mention.

An integration by parts shows that (for $m<n$ )

$$
\begin{aligned}
\left|E X_{m} \bar{X}_{n}\right| & =g_{m} g_{n}\left|\int \widehat{V}_{m}(2 k) \overline{\widehat{V}_{n}(2 k)} e^{2 i\left(\varphi_{m}(k)-\varphi_{n}(k)+k\left(B_{m}-B_{n}\right)\right)} f(k) d k\right| \\
& =\frac{g_{m} g_{n}}{2}\left|\int \frac{\widehat{V}_{m} \widehat{\widehat{V}}_{n} f}{\varphi_{m}^{\prime}-\varphi_{n}^{\prime}+B_{m}-B_{n}} \frac{d}{d k} e^{2 i\left(\varphi_{m}-\varphi_{n}+k\left(B_{m}-B_{n}\right)\right)} d k\right| \\
& \leq \frac{g_{m} g_{n}}{2} \int\left|\frac{d}{d k}\left(\frac{\widehat{V}_{m}(2 k) \overline{\widehat{V}_{n}(2 k)} f(k)}{\varphi_{m}^{\prime}(k)-\varphi_{n}^{\prime}(k)+B_{m}-B_{n}}\right)\right| d k .
\end{aligned}
$$

Evaluating the derivative with the product rule, we get four different terms. The most dangerous term involves the derivative of the denominator. Lemma 2.1 shows that we can estimate this contribution by

$$
C g_{m} g_{n} \sum_{s=1}^{n-1} g_{s} I_{s} \frac{L_{s}^{2}}{L_{n}^{2}} \int\left|\widehat{V}_{m}(2 k) \widehat{V}_{n}(2 k)\right| f(k) d k .
$$

Here and in the sequel, $C$ denotes a constant which depends only on $f$. The actual value of $C$ may change from one formula to the next.

We claim that

$$
\sum_{s=1}^{n-1} g_{s} I_{s} L_{s}^{2} \leq C n^{-1 / 2+\beta / \gamma} L_{n-1}^{2} .
$$

This estimate will be used extensively throughout the rest of this section. It says that the sum is dominated by its last term, and this is true because of the rapid growth of $L_{s}$. Here is the formal argument: Since $L_{n-1} / L_{n} \rightarrow 0$, we can find $C, \alpha>0$ so that $L_{s}^{2} / L_{n-1}^{2} \leq C e^{-\alpha(n-s)}$ for all $s \leq n-1$. Hence

$$
\begin{aligned}
\sum_{s=1}^{n-1} g_{s} I_{s} L_{s}^{2} & \leq C L_{n-1}^{2} e^{-\alpha n} \sum_{s=1}^{n-1} s^{-1 / 2+\beta / \gamma} e^{\alpha s} \\
& \leq C L_{n-1}^{2} e^{-\alpha n} \int_{1}^{n} s^{-1 / 2+\beta / \gamma} e^{\alpha s} d s \leq C n^{-1 / 2+\beta / \gamma} L_{n-1}^{2} .
\end{aligned}
$$

(Recall that $C$ does not necessarily have the same value in every formula.) Here, we have estimated the integral by an integration by parts.

Since supp $f \cap F=\emptyset$, we get from Lemma 3.4a)

$$
\begin{aligned}
\int\left|\widehat{V}_{m} \widehat{V}_{n}\right| f & \leq\left(\int\left|\widehat{V}_{m}\right|^{2} f\right)^{1 / 2}\left(\int\left|\widehat{V}_{n}\right|^{2} f\right)^{1 / 2} \\
& =\left(\int\left|\widehat{V}_{m}-\pi \chi_{F \cup-F} / 2\right|^{2} f \int\left|\widehat{V}_{n}-\pi \chi_{F \cup-F} / 2\right|^{2} f\right)^{1 / 2} \\
& \leq C \epsilon_{m} \epsilon_{n} .
\end{aligned}
$$


Thus we can bound (15) by

$$
C m^{-1 / 2+\beta / \gamma-\beta / 2} n^{-1+2 \beta / \gamma-\beta / 2} \frac{L_{n-1}^{2}}{L_{n}^{2}} .
$$

The other contributions from (14) (where $d / d k$ acts on the numerator) are much easier to deal with. We use the obvious estimates $\left|\widehat{V}_{i}\right| \leq I_{i},\left|\widehat{V}_{i}^{\prime}\right| \leq 2 B_{i} I_{i}$. It follows that these terms can be bounded by $C n^{c} / L_{n}$ for some $c$. In conclusion, we see that $E X_{m} \bar{X}_{n}$ satisfies an estimate of the form

$$
\left|E X_{m} \bar{X}_{n}\right| \leq C\left(\frac{n^{c}}{L_{n}}+m^{-1 / 2+\beta / \gamma-\beta / 2} n^{-1+2 \beta / \gamma-\beta / 2} \frac{L_{n-1}^{2}}{L_{n}^{2}}\right) .
$$

We now assume that the exponent of $m$ is larger than -1 ; the proof in the other case is completely analogous. Then summing over $m$ yields

$$
\sum_{m=1}^{n}\left|E X_{m} \bar{X}_{n}\right| \leq C\left(\frac{n^{c+1}}{L_{n}}+n^{-1 / 2+3 \beta / \gamma-\beta} \frac{L_{n-1}^{2}}{L_{n}^{2}}\right) .
$$

Note that, as promised, the first term on the right-hand side is completely harmless because of the rapid growth of the $L_{n}$ (faster than exponential).

In order to control $E\left|X_{n}\right|^{2}$, we use again the fact that supp $f \cap F=\emptyset$ and apply Lemma 3.4a):

$$
\begin{aligned}
E\left|X_{n}\right|^{2} & =g_{n}^{2} \int\left|\widehat{V}_{n}(2 k)\right|^{2} f(k) d k \\
& =g_{n}^{2} \int\left|\widehat{V}_{n}(2 k)-\pi \chi_{F \cup-F}(k) / 2\right|^{2} f(k) d k \\
& \leq C g_{n}^{2} \epsilon_{n}^{2} \leq C n^{-1+2 \beta / \gamma-\beta} .
\end{aligned}
$$

Now it is straightforward to verify that this estimate and (16) indeed ensure that Proposition 3.2, with

$$
\rho_{n}=C\left(n^{-1+2 \beta / \gamma-\beta}+\frac{n^{c+1}}{L_{n}}+n^{-1 / 2+3 \beta / \gamma-\beta} \frac{L_{n-1}^{2}}{L_{n}^{2}}\right),
$$

applies to $\sum X_{n}$.

In the remainder of this section, it will be convenient to simplify the notation by using the following convention: We will not write out terms which obviously decay so rapidly that they do not pose any difficulties. An example would be the contribution $n^{c+1} / L_{n}$ in (16); note, however, that this term could even be the dominant one in (16), namely, if the $L_{n}$ grow unreasonably fast. In any event, we will alert the reader by writing $\lesssim$ instead of $\leq$ whenever this convention has been applied.

The above strategy can also be used to control $\sum Y_{n}, \sum Z_{n}$. For instance, the leading term in the estimate on $\left|E Y_{m} \bar{Y}_{n}\right|(m<n)$ is

$$
C\left(g_{m} I_{m} g_{n} I_{n}\right)^{2} \sum_{s=1}^{n-1} g_{s} I_{s} \frac{L_{s}^{2}}{L_{n}^{2}} \leq C m^{-1+2 \beta / \gamma} n^{-3 / 2+3 \beta / \gamma} \frac{L_{n-1}^{2}}{L_{n}^{2}}
$$

and summing this over $m(m<n)$ yields the bound $C n^{-3 / 2+5 \beta / \gamma} L_{n-1}^{2} / L_{n}^{2}$. We omit the details of these computations, since the argument is sufficiently close to the discussion above. It turns out that Proposition 3.2 also proves that $\sum Y_{n}, \sum Z_{n}$ are bounded (in fact, convergent) for almost all $k \in \operatorname{supp} f$. 
It remains to show that $\limsup _{N \rightarrow \infty} \sum_{n=1}^{N} g_{n}^{2}\left|\widehat{V}_{n}(2 k)\right|^{2}<\infty$ for almost all $k$ with respect to $d P=f d k$. To this end, let

$$
S(k):=\lim _{N \rightarrow \infty} \sum_{n=1}^{N} g_{n}^{2}\left|\widehat{V}_{n}(2 k)\right|^{2} \in[0, \infty]
$$

Now notice that by monotone convergence and, as above, the fact that supp $f \cap F=$ $\emptyset$ we have that $\int S(k) f(k) d k \leq C \sum g_{n}^{2} \epsilon_{n}^{2}<\infty$, thus indeed $S(k)<\infty$ for almost every $k \in \operatorname{supp} f$. As explained above, this completes the proof of $\sigma_{a c}=[0, \infty)$.

In order to prove the existence of singular continuous spectrum, we use the strategy of the proof of $\left[8\right.$, Theorem 1.6(2)]. So pick $f \in C_{0}^{\infty}(0, \infty)$ with $f \geq 0$, $\int f(k) d k=1$ and $F \subset \operatorname{supp} f$. It suffices to show that (with $\left.d P(k)=f(k) d k\right)$

$$
E\left|\sum_{n=1}^{N} X_{n}\right|^{2}=o\left(\ln ^{2} N\right)
$$

and similarly for $Y_{n}, Z_{n}$. For if this holds, then, by the Chebyshev inequality,

$$
P\left(\left|\sum_{n=1}^{N} X_{n}\right| \geq \delta \ln N\right) \leq \frac{E\left|\sum_{n=1}^{N} X_{n}\right|^{2}}{\delta^{2} \ln ^{2} N} \rightarrow 0 \quad(N \rightarrow \infty)
$$

for any $\delta>0$. In particular, we can find a subsequence $N_{i} \rightarrow \infty$ so that

$$
\sum_{i=1}^{\infty} E\left|\sum_{n=1}^{N_{i}} X_{n}\right|^{2} \ln ^{-2} N_{i}<\infty
$$

and now the Borel-Cantelli Lemma says that

$$
P\left(\left|\sum_{n=1}^{N_{i}} X_{n}\right| \geq \delta \ln N_{i} \text { for infinitely many } i\right)=0 .
$$

Of course, analogous statements hold if $X_{n}$ is replaced with $Y_{n}$ or $Z_{n}$.

On the other hand, Lemma 3.4b) implies that $\lim _{n \rightarrow \infty} \widehat{V}_{n}(2 k)=\pi / 2$ for almost every $k \in F$. If $\delta$ from above is small enough, we thus see from (8) that

$$
\lim _{i \rightarrow \infty} R_{N_{i}}(k) \geq \lim _{i \rightarrow \infty}\left(c_{1} \sum_{n=1}^{N_{i}} g_{n}^{2}-c_{2} \delta \ln N_{i}\right)=\infty
$$

for almost every $k \in F$. Hence the absolutely continuous part of the spectral measure $\rho_{\alpha}$ gives zero weight to $F^{2}=\left\{k^{2}: k \in F\right\}$ [10, Theorem 1.2]. As noted at the beginning of the proof, this also holds for the point part of $\rho_{\alpha}$. Moreover, $\alpha$ was arbitrary, so the spectral averaging formula (see e.g. [3]) becomes

$$
0<\left|F^{2}\right|=\int_{0}^{\pi} \rho_{\alpha}\left(F^{2}\right) d \alpha=\int_{0}^{\pi} \rho_{\alpha}^{(s c)}\left(F^{2}\right) d \alpha .
$$

This forces $\rho_{\alpha}^{(s c)}\left(F^{2}\right)>0$ for a set of $\alpha$ 's of positive measure, as desired.

It remains to prove (17). To this end, let $S_{N}=\sum_{n=1}^{N} X_{n}$. Then

$$
E\left|S_{N}\right|^{2} \leq E\left|S_{N-1}\right|^{2}+E\left|X_{N}\right|^{2}+2\left|E S_{N-1} \bar{X}_{N}\right|
$$


Clearly, $E\left|X_{N}\right|^{2} \leq C g_{N}^{2}=C N^{-1}$. In order to estimate $E S_{N-1} \bar{X}_{N}$, we use the integration by parts argument from the first part of this proof. This gives

$$
\left|E S_{N-1} \bar{X}_{N}\right| \leq C g_{N} \int\left|\frac{d}{d k}\left(\frac{f(k) \overline{\widehat{V}_{N}(2 k)} S_{N-1}(k)}{\varphi_{N}^{\prime}(k)+B_{N}}\right)\right| d k .
$$

This time, there are two potentially dangerous terms: the first one coming from the derivative of the denominator and the second one involving $d S_{N-1} / d k$. The first contribution is treated as above to obtain the bound

$$
C g_{N} \sum_{s=1}^{N-1} g_{s} I_{s} \frac{L_{s}^{2}}{L_{N}^{2}} \int\left|f \widehat{V}_{N} S_{N-1}\right| d k \leq C N^{-1+\beta / \gamma} \frac{L_{N-1}^{2}}{L_{N}^{2}}\left(E\left|S_{N-1}\right|^{2}\right)^{1 / 2} .
$$

The last estimate follows by the Cauchy-Schwarz inequality, Lemma 3.4a), and the usual bound on $\sum_{s=1}^{N-1} g_{s} I_{s} L_{s}^{2}$.

As for the second term, we note that the leading term of

$$
\frac{d S_{N-1}}{d k}=\sum_{n=1}^{N-1} g_{n} \frac{d}{d k} \widehat{V}_{n}(2 k) e^{2 i\left(\varphi_{n}(k)+k B_{n}\right)}
$$

comes from differentiating $e^{2 i \varphi_{n}}$. Now the usual techniques show that the corresponding contribution to $E S_{N-1} \bar{X}_{N}$ can be bounded by $C g_{N}^{2} L_{N-1} / L_{N}$.

As before, we need not worry about the remaining terms which can be bounded by an expression of the form $C N^{c} / L_{N}$, and the rapid growth of $L_{N}$ guarantees that these terms are unimportant. So, if we put everything together, (18) becomes

$$
E\left|S_{N}\right|^{2} \lesssim E\left|S_{N-1}\right|^{2}+C N^{-1}+C N^{-1+\beta / \gamma} \frac{L_{N-1}^{2}}{L_{N}^{2}}\left(E\left|S_{N-1}\right|^{2}\right)^{1 / 2} .
$$

By an inductive argument, one can now prove that

$$
\left(E\left|S_{N}\right|^{2}\right)^{1 / 2} \lesssim C \sum_{n=1}^{N} n^{-1+\beta / \gamma} \frac{L_{n-1}^{2}}{L_{n}^{2}}+C\left(\sum_{n=1}^{N} n^{-1}\right)^{1 / 2} .
$$

In fact, the statement needed here is exactly [8, Lemma 6.2]; it would be pointless to repeat that proof here. In any event, using the assumptions of Theorem 3.3, we see that we have the required bound $E\left|S_{N}\right|^{2}=o\left(\ln ^{2} N\right)$.

The proof of (17) for $Y_{n}$ and $Z_{n}$ is similar. Again, we sketch the argument for $Y_{n}$ and leave the proof for $Z_{n}$ to the reader.

An elementary estimate yields $E\left|Y_{N}\right|^{2} \leq C N^{-2+4 \beta / \gamma}$. As usual, $E S_{N-1} \bar{Y}_{N}$ (here, $S_{n}=\sum_{t=1}^{n} Y_{t}$, of course) is treated with an integration by parts, and the most serious attention has to be paid to the contribution involving $(d / d k)\left(1 /\left(\varphi_{N}^{\prime}+B_{N}\right)\right)$. This term can be bounded by

$$
C\left(g_{N} I_{N}\right)^{2} \sum_{s=1}^{N-1} g_{s} I_{s} \frac{L_{s}^{2}}{L_{N}^{2}} \int f\left|S_{N-1}\right| d k \leq C N^{-3 / 2+3 \beta / \gamma} \frac{L_{N-1}^{2}}{L_{N}^{2}}\left(E\left|S_{N-1}\right|^{2}\right)^{1 / 2} .
$$

If we collect all terms and use the inductive argument from above, we finally get the following estimate

$$
\left(E\left|S_{N}\right|^{2}\right)^{1 / 2} \lesssim C \sum_{n=1}^{N} n^{-3 / 2+3 \beta / \gamma} \frac{L_{n-1}^{2}}{L_{n}^{2}}+C\left(\sum_{n=1}^{N} n^{-2+4 \beta / \gamma}\right)^{1 / 2} .
$$


Again, a routine verification shows that this latter expression is of order $o(\ln N)$ (in fact, it is even bounded), as desired.

One can interchange the roles of $F$ and $(0, \infty) \backslash F$ to obtain potentials with embedded absolutely continuous spectrum. More precisely, proceed as follows: Pick an even function $g \in \mathcal{S}(\mathbb{R})$ (the Schwartz class) with $g>0$ and $g(k)=1$ if $k \in F$. Define

$$
W(x)=\int_{0}^{\infty}\left(g(k)-\chi_{F}(k)\right) \cos 2 k x d k .
$$

Theorem 3.5. Assume that $F, \gamma, g_{n}, B_{n}, L_{n}$ satisfy the assumptions of Theorem 3.3. Then, for all $\alpha$, the half-line Schrödinger operator $H_{\alpha}$ with potential $V$ given by (2), (10), (19) satisfies $\sigma_{s c}\left(H_{\alpha}\right)=\sigma_{\text {ess }}\left(H_{\alpha}\right)=[0, \infty), \sigma_{p}\left(H_{\alpha}\right) \cap(0, \infty)=\emptyset$ and $\sigma_{a c}\left(H_{\alpha}\right)=F^{2}$.

Large parts of this proof are similar to the corresponding arguments of the proof of Theorem 3.3. Therefore, these parts of the proof will only be sketched.

As before, it is easy to verify the assertions on $\sigma_{e s s}, \sigma_{p}$. We also have an analogue of Lemma 3.4 where the function $\pi \chi_{F \cup-F}(k) / 2$ now is replaced with $\widehat{W}(k):=$ $\pi\left(g(k)-\chi_{F \cup-F}(k)\right) / 2$. Note that $\widehat{W}(k)=0$ if $k \in F$. Moreover, if $I \subset(0, \infty)$ is a compact set with $I \cap F=\emptyset$, then $\inf _{k \in I}|\widehat{W}(k)|>0$. Finally, we still have the estimates (13), because $g \in \mathcal{S}(\mathbb{R})$ implies that the part $\int g(k) \cos 2 k x d k$ of $W$ decays faster than any power of $x$.

To prove the assertion on $\sigma_{s c}$, fix $f \in C_{0}^{\infty}(0, \infty)$ with $f \geq 0$, supp $f \cap F=\emptyset$, and $\int f d k=1$. Now we can repeat the arguments from the last part of the proof of Theorem 3.3. In this way, we see that $\rho_{a c}^{(\alpha)}\left((0, \infty) \backslash F^{2}\right)=0$ for all $\alpha$, hence, since $F$ is nowhere dense and $\sigma_{\text {ess }}=[0, \infty), \sigma_{p} \cap(0, \infty)=\emptyset$, we must have $\sigma_{s c}\left(H_{\alpha}\right)=[0, \infty)$, as claimed.

Now let $d P(k)=|F|^{-1} \chi_{F}(k) d k$. We want to estimate $E X_{m} \bar{X}_{n}(m \leq n)$, where the expectation is computed with this probability measure. To this end, we first observe that there exist functions $f_{N}(k) \in C_{0}^{\infty}(0, \infty)(N \in \mathbb{N})$ with $0 \leq f_{N} \leq 1$ and

$$
\int\left|\chi_{F}(k)-f_{N}(k)\right| d k \leq C 2^{(1-\gamma) N}, \quad \int\left|f_{N}^{\prime}(k)\right| d k=2^{N+1}
$$

( $\gamma$ is from Lemma 3.1). To see this, simply approximate $\chi_{F_{N}}$ by an appropriate smooth function. Here, $F_{N}$ is the set obtained in the $N$ th step of the construction of $F$ (see the discussion preceding Lemma 3.1).

Using these approximations of $\chi_{F}$, we get $($ for $m<n$ )

$$
\begin{aligned}
\left|E X_{m} \bar{X}_{n}\right| & =g_{m} g_{n}|F|^{-1}\left|\int \chi_{F}(k) \widehat{V}_{m}(2 k) \overline{\widehat{V}_{n}(2 k)} e^{2 i\left(\varphi_{m}(k)-\varphi_{n}(k)+k\left(B_{m}-B_{n}\right)\right)} d k\right| \\
\leq & g_{m} g_{n}|F|^{-1}\left(I_{m} I_{n} \int\left|\chi_{F}(k)-f_{N}(k)\right| d k\right. \\
& \left.+\left|\int f_{N}(k) \widehat{V}_{m}(2 k) \widehat{\widehat{V}}_{n}(2 k) e^{2 i\left(\varphi_{m}(k)-\varphi_{n}(k)+k\left(B_{m}-B_{n}\right)\right)} d k\right|\right) .
\end{aligned}
$$

Clearly, the first term can be bounded by $C g_{m} g_{n} I_{m} I_{n} 2^{(1-\gamma) N}$. In the second term, we use again an integration by parts. This time, the terms obtained by differentiating $f_{N}$ and, as usual, $1 /\left(\varphi_{m}^{\prime}-\varphi_{n}^{\prime}+B_{m}-B_{n}\right)$ deserve the most serious attention. We use the by now familiar procedures and also (20) for the term involving $f_{N}^{\prime}$. We 
get bounds of the form $C g_{m} g_{n} 2^{N} I_{m} I_{n} / L_{n}$ and

$$
\begin{aligned}
& C g_{m} g_{n} n^{-1 / 2+\beta / \gamma} \frac{L_{n-1}^{2}}{L_{n}^{2}} \int f_{N}\left|\widehat{V}_{m} \widehat{V}_{n}\right| d k \\
& \leq C m^{-1 / 2} n^{-1+\beta / \gamma} \frac{L_{n-1}^{2}}{L_{n}^{2}}\left(I_{m} I_{n} \int\left|f_{N}-\chi_{F}\right| d k+\int \chi_{F}\left|\widehat{V}_{m} \widehat{V}_{n}\right| d k\right) \\
& \leq C m^{-1 / 2} n^{-1+\beta / \gamma} \frac{L_{n-1}^{2}}{L_{n}^{2}}\left((m n)^{\beta / \gamma} 2^{(1-\gamma) N}+\epsilon_{m} \epsilon_{n}\right),
\end{aligned}
$$

respectively. Now we can take, say, $N=n$ and sum the resulting bounds over $m$ (again, we will treat explicitly only the case where the exponent of $m$ is larger than $-1)$. We thus obtain

$$
\sum_{m=1}^{n-1}\left|E X_{m} \bar{X}_{n}\right| \lesssim C n^{-1 / 2+3 \beta / \gamma-\beta} \frac{L_{n-1}^{2}}{L_{n}^{2}} .
$$

Moreover, the analogue of Lemma 3.4a) implies that $E\left|X_{n}\right|^{2}=g_{n}^{2}|F|^{-1} \int_{F}\left|\widehat{V}_{n}(2 k)\right|^{2}$ $d k \leq C g_{n}^{2} \epsilon_{n}^{2}$. These estimates together with the assumptions of the theorem show that Proposition 3.2 is applicable. By similar arguments, the same holds for $Y_{n}$ and $Z_{n}$.

Finally, by the analogue of Lemma 3.4a) again,

$$
\sum_{n=1}^{\infty} g_{n}^{2} \int_{F}\left|\widehat{V}_{n}(2 k)\right|^{2} d k \leq C \sum_{n=1}^{\infty} g_{n}^{2} \epsilon_{n}^{2}<\infty
$$

so the monotone convergence theorem implies that $\sum g_{n}^{2}\left|\widehat{V}_{n}(2 k)\right|^{2}<\infty$ for almost every $k \in F$. We have thus shown that for almost all $k \in F,(1)$ has only bounded solutions. Therefore every subset $F^{\prime} \subset F$ with $\left|F^{\prime}\right|>0$ also satisfies $\rho_{a c}^{(\alpha)}\left(F^{\prime 2}\right)>0$ [23, Theorem 5]. It is easy to see from the properties of $F$ that this forces $\sigma_{a c} \supset F^{2}$. On the other hand, we know already that $\sigma_{a c} \subset F^{2}$, hence $\sigma_{a c}=F^{2}$, as claimed.

\section{Subordinate Solutions}

To begin with, recall the results from [4]. Write $\|y\|_{x}:=\left(\int_{0}^{x}|y(t)|^{2} d t\right)^{1 / 2}$. A solution $y \not \equiv 0$ of (1) is called subordinate if $\lim _{x \rightarrow \infty}\|y\|_{x} /\|w\|_{x}=0$ for every linearly independent solution $w$ of the same equation. The generalized eigenfunction $v_{\alpha}(x, E)$ is, by definition, the solution of (1) with the initial values $v_{\alpha}(0, E)=$ $-\sin \alpha, v_{\alpha}^{\prime}(0, E)=\cos \alpha$. Note that $v_{\alpha}$ satisfies the boundary condition described by $\alpha$.

The following basic result relates the notion of subordinacy to the boundary behavior of the $m$-function (for more information on the $m$-function, see e.g. [1]).

Theorem $4.1([4]) \cdot v_{\alpha}(\cdot, E)$ is subordinate if and only if $\lim _{\epsilon \rightarrow 0+}\left|m_{\alpha}(E+i \epsilon)\right|=$ $\infty$.

Motivated by Theorem 4.1, we consider the sets

$$
S_{\alpha}=\left\{E \in \mathbb{R}: v_{\alpha}(\cdot, E) \text { is subordinate }\right\} .
$$

Then, by Theorem 4.1 and basic facts on the $m$-function, $S_{\alpha}$ supports the singular part of the spectral measure $\rho_{\alpha}$ (see [4]). Note that each $S_{\alpha}$ has Lebesgue measure zero because the $m$-function has a finite limit almost everywhere. Therefore, the 
result below is of interest. It shows that $S_{\alpha}$ can be large without there being any singular continuous spectrum (namely, if $g_{n} \in l_{2} \backslash l_{1}$ in Theorem 4.2).

These phenomena can not occur for point or absolutely continuous spectra. That is to say, the existence (or non-existence) of point and absolutely continuous spectrum, respectively, can always be read off from the size of the corresponding minimal supports. This is due to the fact that point and absolutely continuous measures are equivalent to counting and Lebesgue measure, respectively, restricted to these supports. Singular continuous measures do not, in general, have such a universal property. See also [2], [9] for a discussion of related issues in the context of Schrödinger operators.

Theorem 4.2. Assume that $B_{n}=B, V_{n}(x)=W(x)$ (where $W \in L_{1}([-B, B])$, $W \not \equiv 0), g_{n} \rightarrow 0$, and $L_{n-1} / L_{n} \rightarrow 0$. Consider the Schrödinger operators $H_{\alpha}$ with potential $V$ given by (2). Then for all boundary conditions $\alpha$, we have:

a) [8] If $g_{n} \in l_{2}$, then the spectrum of $H_{\alpha}$ is purely absolutely continuous on $(0, \infty)$. b) If $g_{n} \notin l_{1}$, then for any open set $U \subset(0, \infty)$, the set $S_{\alpha} \cap U$ has Hausdorff dimension 1 .

Remarks. 1. We could also treat $n$-dependent barriers $V_{n}$ with our methods, but in this case one needs additional assumptions on the Fourier transforms $\widehat{V}_{n}$.

2. If $g_{n} \notin l_{2}$, the spectrum is purely singular continuous on $(0, \infty)$ by $[8$, Theorem 1.6(2)]. Note that part b) of the theorem continues to hold. However, in this case this statement follows quite easily from the results of [6], [15], and the following rather involved proof would clearly be inappropriate here.

Proof. a) This is [8, Theorem 1.6(1)].

b) Here is the strategy of the proof. Fix $\alpha$, and assume, without loss of generality, that $U$ is contained in a compact subset of $(0, \infty)$. Also, as above, it will be convenient to work with wavenumbers $k=\sqrt{E}$ instead of energies $E$. Since $I_{n}$ is constant, (7) yields

$$
\ln R_{N+1}(k)=\frac{1}{2 k} \operatorname{Im} \widehat{W}(2 k) \sum_{n=1}^{N} g_{n} e^{2 i \varphi_{n}(k)}+\rho_{N}(k)
$$

where $\left|\rho_{N}(k)\right| \leq C(U) \sum_{n=1}^{N} g_{n}^{2}$ for all $k \in U$ and

$$
\widehat{W}(2 k)=\int_{-B}^{B} W(x) e^{2 i k(x+B)} d x .
$$

We will construct a probability measure $P$ on $U$ so that the sum on the right-hand side of (21) goes to $-\infty$ almost surely with respect to $P$. Moreover, $P$ will give zero weight to sets of Hausdorff dimension less than 1. In this way, we will obtain "small" solutions on a set of Hausdorff dimension 1.

To carry out this program, pick numbers $N_{n} \in \mathbb{N}$, such that $N_{n} \rightarrow \infty$ and $N_{n} N_{n-1} L_{n-1} / L_{n} \rightarrow 0$ as $n \rightarrow \infty$. Since $\widehat{W}$ is analytic, its zeros are isolated. Thus it clearly suffices to prove the claim for the case when $U$ is an open interval with $\inf _{k \in U}|\widehat{W}(2 k)|>0$ (and, as above, $\inf U>0, \sup U<\infty$ ). For $n \geq n_{0}$, we let

$$
l_{n}:=\frac{\pi}{N_{n} L_{n}}\left(1+\frac{\theta_{n}}{N_{n}}\right)
$$

with as yet unspecified $n_{0} \in \mathbb{N}$ and $\theta_{n} \in[0,1]$. We also set $l_{n_{0}-1}=|U|$. Now a straightforward computation shows that it is possible to choose first $n_{0}$ sufficiently 
large and then inductively the $\theta_{n}\left(n \geq n_{0}\right)$, such that

$$
r_{n}:=\frac{l_{n-1}}{l_{n} N_{n}} \in \mathbb{N} \quad \forall n \geq n_{0} .
$$

For instance, we have that for $n>n_{0}$ and for fixed $\theta_{n-1}$ the quantity

$$
\frac{l_{n-1}}{l_{n} N_{n}}=\frac{L_{n}}{N_{n-1} L_{n-1}} \frac{1+\theta_{n-1} / N_{n-1}}{1+\theta_{n} / N_{n}}
$$

runs over an interval of size $\geq L_{n} /\left(L_{n-1} N_{n-1}\left(N_{n}+1\right)\right)$ as $\theta_{n}$ runs over $[0,1]$. Since this latter expression tends to infinity, the corresponding interval must contain an integer, provided $n$ is large enough. A similar argument works in the case $n=n_{0}$.

In order to simplify the notation, we will assume that $n_{0}=1$. The reader can verify easily that our arguments are valid in the general case as well.

By definition of $r_{1}$ and $l_{0}$, the original interval $U=I^{(0)}$ can be divided into $r_{1} N_{1}$ subintervals $I_{i}^{(1)}$ of equal length $l_{1}$. Each of these subintervals $I_{i}^{(1)}$, in turn, can be divided into $r_{2} N_{2}$ sub-subintervals $I_{j}^{(2)}$ of equal length $l_{2}$ etc. So we obtain a sequence of partitions $U=\bigcup_{j} I_{j}^{(n)}$ which become increasingly finer. Every interval $I_{j}^{(n)}$ belonging to the $n$th partition has length $l_{n}$ and contains exactly $r_{n+1} N_{n+1}$ elements of the $(n+1)$ st partition.

Now we define correspondingly discrete approximations $\psi_{n}$ of the Prüfer angles $\varphi_{n}$. The variable $\psi_{n}$ will take the values $t \pi / N_{n}\left(t=0,1, \ldots, N_{n}-1\right)$, and $\psi_{n}$ will be constant on every $I_{j}^{(n)}$. Let $n \in \mathbb{N}$, fix one of the intervals $I_{i}^{(n-1)}$, and consider the corresponding subintervals $I_{j}^{(n)} \subset I_{i}^{(n-1)}$. By construction, there are $r_{n} N_{n}$ such intervals $I_{j}^{(n)}$. To fix the notation, let us assume that we obtain these intervals if $j$ runs from 1 to $r_{n} N_{n}$. We further assume that this labeling is the natural one in the sense that if $j<j^{\prime}$, then $I_{j}^{(n)}$ lies left of $I_{j^{\prime}}^{(n)}$. Now consider the $s$ th group of $N_{n}$ such intervals (where $s \in\left\{1, \ldots, r_{n}\right\}$ ), i.e. consider

$$
\left\{I_{j}^{(n)}: j=(s-1) N_{n}+1, \ldots, s N_{n}\right\} .
$$

Determine $t_{0} \in\left\{0,1, \ldots, N_{n}-1\right\}$ so that $\left|t_{0} \pi / N_{n}-\varphi_{n}\left(k_{0}\right)\right|$ is minimal. In this definition, $k_{0}$ is the left endpoint of the first interval (i.e. $j=(s-1) N_{n}+1$ in (23)), and the difference $t_{0} \pi / N_{n}-\varphi_{n}\left(k_{0}\right)$ has to be evaluated modulo $\pi$. Set $\psi_{n}(k)=\left(t_{0}-1+m\right) \pi / N_{n}$ if $k$ lies in the $m$ th interval (i.e. $j=(s-1) N_{n}+m$ in (23)). Here, $m$ takes the values $m=1, \ldots, N_{n}$. Carry out this procedure for all $I_{i}^{(n-1)}$ to define $\psi_{n}(k)$ on all of $k \in U$.

In fact, strictly speaking, this does not define $\psi_{n}$ on all of $U$, since we have not distinguished between the open and closed intervals $I_{j}^{(n)}$. So the reader might worry (rightly, at this point) that $\psi_{n}(k)$ is not well-defined at the endpoints of the $I_{j}^{(n)}$. However, as we will see shortly, the measure $P$ we are going to construct will be continuous, and thus the above procedure does define $\psi_{n}(k)$ almost everywhere. This is all we will need. For definiteness, let us agree that in the sequel, it is understood that $I_{j}^{(n)}$ denotes the open interval.

Note that, by Lemma 2.1,

$$
\varphi_{n}(k+l)-\varphi_{n}(k)=l L_{n}\left(1+O\left(L_{n-1} / L_{n}\right)\right) .
$$


Using this, (22), and $N_{n} L_{n-1} / L_{n} \rightarrow 0$, one can verify easily that

$$
\left|\psi_{n}(k)-\varphi_{n}(k)\right| \leq \frac{C}{N_{n}}
$$

where $C$ depends only on $U$.

Now let $p_{n}(t)$ be probabilities, i.e. we require that $p_{n}(t) \geq 0$ and $\sum_{t=0}^{N_{n}-1} p_{n}(t)=1$ for all $n$. Notice that, by construction, $\psi_{m}(k)$ is constant on every interval $k \in I_{j}^{(n)}$ with $n \geq m$ because every such interval is contained in some $I_{i}^{(m)}$. Thus it makes sense to look for a measure $P$ with the property that for all $I_{j}^{(n)}$,

$$
P\left(I_{j}^{(n)}\right)=\prod_{m=1}^{n} \frac{p_{m}\left(t_{m}\right)}{r_{m}}
$$

where the $t_{m}$ describe the values of the $\psi_{m}$ on $I_{j}^{(n)}$. More precisely, $t_{m} \in\{0,1, \ldots$, $\left.N_{m}-1\right\}$ is defined by requiring that the value of $\psi_{m}$ on $I_{j}^{(n)}$ be given by $t_{m} \pi / N_{m}$.

Lemma 4.3. a) There is a unique probability (Borel) measure $P$ on $I^{(0)}=U$, such that $P\left(I_{j}^{(n)}\right)$ is given by (25) for all $n, j$.

b) The $\psi_{n}$ become independent random variables with distributions $p_{n}$, i.e.

$$
P\left(\psi_{1}=\frac{t_{1} \pi}{N_{1}}, \ldots, \psi_{n}=\frac{t_{n} \pi}{N_{n}}\right)=p_{1}\left(t_{1}\right) \cdots p_{n}\left(t_{n}\right) .
$$

c) Suppose $p_{n}(t) \leq C / N_{n}$ for all $n, t$. Then $P(A)=0$ for every set $A \subset U$ with Hausdorff dimension $\operatorname{dim} A<1$.

Remark. We gave the lemma in a slightly more general form than actually needed here (for instance, we do not need uniqueness) because it is easy to obtain these stronger statements.

Proof. a) To begin with, note that any $P$ satisfying (25) must be continuous: On the one hand, for any fixed $n$, the measures of the (open!) intervals $I_{j}^{(n)}$ already add up to 1 , hence the endpoints of the $I_{j}^{(n)}$ are a set of measure zero. On the other hand, since $r_{n} \rightarrow \infty$, there cannot be any atoms different from these endpoints, either.

Now let $P_{n}$ be the right continuous (say), monotonically increasing step function with the following properties: $P_{n}(\inf U)=0$, and $P_{n}$ is constant on every $I_{j}^{(n)}$ and has a jump of size $P\left(I_{j}^{(n)}\right)$ at the right endpoint of $I_{j}^{(n)}$. It is easy to verify that the $P_{n}$ increase to a limit function $P$ which generates a probability measure consistent with (25). This proves existence.

For the uniqueness part, we need only show that (25) determines $P(a, b)$ for arbitrary $a<b$ (since we know already that $P$ is continuous). This, however, is obvious, because any open interval $(a, b)$ can be approximated by an increasing sequence of finite unions of the original intervals $I_{j}^{(n)}$.

b) This follows from (25). Note that by construction the conditions $\psi_{i}=$ $t_{i} \pi / N_{i}(i=1, \ldots, n)$ hold on precisely $r_{1} \cdots r_{n}$ intervals $I_{j}^{(n)}$.

c) By general facts on Hausdorff measures [18, Section 3.3], the assertion will follow if we can show that for any fixed $\gamma<1$, we have that for $P$-almost every $k$,

$$
\lim _{\delta \rightarrow 0+} \sup _{J} \frac{P(J)}{|J|^{\gamma}}=0 .
$$


Here, the sup is taken over all intervals $J$ of length $|J| \leq \delta$ which contain $k$. In fact, we will show that in the case at hand, (26) holds for all $k \in U$, and the limit is uniform in $k$.

So let $J \subset U$ be an interval of length $|J| \leq \delta$. Let $n$ be the largest integer for which $J$ is contained in the union of two intervals $I_{j}^{(n)} \cup I_{j+1}^{(n)}$. Then $J$ contains at least one of the intervals $I_{i}^{(n+1)}$ (for if this is not true, we get a contradiction to the definition of $n$ ). In particular, $2 \leq 2|J| / l_{n+1}$, and thus $J$ intersects at most $2+\left(|J| / l_{n+1}\right) \leq 3|J| / l_{n+1}$ intervals $I_{i}^{(n+1)}$. Clearly, each of these intervals satisfies

$$
P\left(I_{i}^{(n+1)}\right) \leq \frac{C}{r_{n+1} N_{n+1}} \max _{j} P\left(I_{j}^{(n)}\right)
$$

and therefore,

$$
P(J) \leq \frac{3 C|J|}{r_{n+1} N_{n+1} l_{n+1}} \max _{j} P\left(I_{j}^{(n)}\right)=\frac{3 C|J|}{l_{n}} \max _{j} P\left(I_{j}^{(n)}\right) .
$$

Using $|J| \leq 2 l_{n}$, the definitions of $r_{n}, l_{n}$, and the obvious estimate $\max P\left(I_{j}^{(n)}\right) \leq$ $C^{n} \prod_{m=1}^{n}\left(r_{m} N_{m}\right)^{-1}$, we deduce that for fixed $\gamma<1$,

$$
\frac{P(J)}{|J|^{\gamma}} \leq \frac{12 \pi C^{n+1}}{l_{0}}\left(N_{n} L_{n}\right)^{\gamma-1}
$$

Since $L_{n}$ grows faster than exponentially (recall $L_{n} / L_{n-1} \rightarrow \infty$ ) and $n$ goes to infinity as $\delta$ tends to zero, we see that we indeed have (26).

As our next step in the proof of Theorem 4.2, we note

Lemma 4.4. Let $P$ be as in Lemma 4.3. Then for P-almost every $k$ we have that

$$
\ln R_{N+1}(k)=\frac{1}{2 k} \operatorname{Im} \widehat{W}(2 k) \sum_{n=1}^{N} g_{n} E\left(e^{2 i \psi_{n}}\right)+o\left(\sum_{n=1}^{N} g_{n}\right) .
$$

Remarks. 1. There is an analogue of this lemma for $n$-dependent barriers $V_{n}$. The proof is more difficult in this case.

2. The estimate on the error term is not uniform in $k$.

Proof. If we replace the angles $\varphi_{n}$ in (21) with their discrete counterparts $\psi_{n}$, then, by $(24)$, we obtain an error of order

$$
O\left(\sum_{n=1}^{N} g_{n} / N_{n}\right)=o\left(\sum_{n=1}^{N} g_{n}\right)
$$

$\left(\right.$ recall $\left.N_{n} \rightarrow \infty\right)$. It remains to show that, almost surely, $\sum_{n=1}^{N} X_{n}=o\left(\sum_{n=1}^{N} g_{n}\right)$ where $X_{n}:=g_{n}\left(e^{2 i \psi_{n}}-E\left(e^{2 i \psi_{n}}\right)\right)$. By Lemma $\left.4.3 \mathrm{~b}\right)$, the $X_{n}$ are independent random variables. Moreover, $E X_{n}=0$ and $E\left|X_{n}\right|^{2} \leq g_{n}^{2}$, hence, by [24, Theorem 3.3.1] with $p=2$, it suffices to show that $\sum g_{n}^{2}\left(\sum_{i=1}^{n} g_{i}\right)^{-2}<\infty$. This, however, is 
easy to check:

$$
\begin{aligned}
\sum_{n=2}^{\infty} \frac{g_{n}^{2}}{\left(\sum_{i=1}^{n} g_{i}\right)^{2}} & \leq C \sum_{n=2}^{\infty} \frac{g_{n}}{\left(\sum_{i=1}^{n} g_{i}\right)^{2}} \\
& =C \sum_{n=2}^{\infty}\left(1-\frac{g_{n}}{\sum_{i=1}^{n} g_{i}}\right)\left(\frac{1}{\sum_{i=1}^{n-1} g_{i}}-\frac{1}{\sum_{i=1}^{n} g_{i}}\right) \\
& \leq C \sum_{n=2}^{\infty}\left(\frac{1}{\sum_{i=1}^{n-1} g_{i}}-\frac{1}{\sum_{i=1}^{n} g_{i}}\right)<\infty
\end{aligned}
$$

Now we are at last in a position to prove the claim of the theorem. First of all, recall the remarks made at the beginning of this proof. So, let $U \subset(0, \infty)$ be an open interval with $\inf U>0, \sup U<\infty$, and $\inf _{k \in U}|\widehat{W}(2 k)|>0$. We further demand that the phase of $\widehat{W}$ does not vary much if $k$ runs over $U$. More precisely, write $\widehat{W}(2 k)=|\widehat{W}(2 k)| e^{i \beta(2 k)}$; we suppose that

$$
\sup _{k, k^{\prime} \in U}\left|\beta(2 k)-\beta\left(2 k^{\prime}\right)\right| \leq \pi / 8
$$

This, of course, can be ensured by taking $U$ sufficiently small.

Next, we want to use Lemma 4.3 to construct appropriate probability measures on $U$. Thus we need to specify the $p_{n}(t)$ from (25). To this end, first choose $t_{0}(n) \in\left\{0,1, \ldots, N_{n}-1\right\}$ such that

$$
\left|\frac{2 t_{0}(n) \pi}{N_{n}}+\beta(2 k)+\pi\right| \leq \frac{\pi}{4}
$$

for all $k \in U$ (the left-hand side of (28) is to be evaluated modulo $2 \pi$ ). This is possible by (27), at least if we assume, without loss of generality, that $N_{n} \geq 8$. We choose the $N_{n}$ as even numbers and set

$$
p_{n}\left(t_{0}(n)\right)=\ldots=p_{n}\left(t_{0}(n)-1+N_{n} / 2\right)=2 / N_{n},
$$

and $p_{n}(t)=0$ otherwise. (In this definition, the argument of $p_{n}$ has to be evaluated modulo $N_{n}$.) Now, Lemma 4.3 yields a probability measure $P$, such that the $\psi_{n}$ are independent with distributions determined by the $p_{n}(t)$, according to Lemma $4.3 \mathrm{~b})$.

We compute

$$
\begin{aligned}
E\left(e^{2 i \psi_{n}}\right) & =\frac{2}{N_{n}} \sum_{t=t_{0}(n)}^{t_{0}(n)-1+N_{n} / 2} e^{2 \pi i t / N_{n}}=-\frac{4}{N_{n}} \frac{e^{2 \pi i t_{0}(n) / N_{n}}}{e^{2 \pi i / N_{n}}-1} \\
& =\frac{2 i}{\pi} e^{2 \pi i t_{0}(n) / N_{n}}+O\left(N_{n}^{-1}\right) .
\end{aligned}
$$

Note that by (28), we have $\operatorname{Im} i e^{2 \pi i t_{0}(n) / N_{n}} e^{i \beta(2 k)} \leq-1 / \sqrt{2}$ for all $n \in \mathbb{N}, k \in U$. Thus by combining Lemma 4.3c) and Lemma 4.4 we see that on a $k$-set of Hausdorff dimension 1, the Prüfer radius $R$ satisfies

$$
\ln R_{N+1}(k) \leq-c \sum_{n=1}^{N} g_{n}
$$

for all large enough $N \geq N_{0}=N_{0}(k)$. The constant $c>0$ depends only on $U$. If we consider the corresponding set of energies $E=k^{2}$, we still have a set of Hausdorff dimension 1 because the map $k \rightarrow E=k^{2}$ is bi-Lipschitz on every compact 
subset of $(0, \infty)$. Recall that $R$ is given by $R(x, k)^{2}=v_{\alpha}(x, k)^{2}+v_{\alpha}^{\prime}(x, k)^{2} / k^{2}$ (cf. Section 2) where $v_{\alpha}$ is the generalized eigenfunction introduced at the beginning of this section. Now let $y$ be any solution of the Schrödinger equation (1) which is linearly independent of $v_{\alpha}(\cdot, k)$, and let $\tilde{R}^{2}=y^{2}+y^{\prime 2} / k^{2}$ and $\tilde{\varphi}$ be the corresponding Prüfer variables. Constancy of the Wronskian $W\left(v_{\alpha}, y\right)=v_{\alpha} y^{\prime}-v_{\alpha}^{\prime} y$ yields $R \tilde{R} \sin (\varphi-\tilde{\varphi})=w \neq 0$, hence $\tilde{R} \geq|w| / R$. It is easy to see that for the potentials under consideration, one has inequalities of the type $\|y\|_{x}^{2} \geq c \int_{0}^{x} \tilde{R}^{2}$ (with $c>0$, of course) for all sufficiently large $x$. Hence (29) together with the estimate $\tilde{R} \geq|w| / R$ guarantee that the solution $v_{\alpha}$ is subordinate.

The methods of this proof clearly extend to more general Hausdorff measures. More specifically, let $h(t)$ be an increasing, right continuous function on $[0, \infty)$ with $h(0)=0$ and $h(t)>0$ for $t>0$. Then one can define a (generalized) Hausdorff measure $\mu_{h}$ (see [18, Section 2.1]; the details are not of interest here). The usual $\gamma$ dimensional Hausdorff measures are obtained with the choice $h(t)=t^{\gamma}$. Now only a minor modification in the proof of Lemma 4.3c) is needed to prove the following result: Let $h$ be as above with $\lim _{t \rightarrow 0+} h(t) / t=\infty$. If the $L_{n}$ grow sufficiently rapidly (this condition can be put in a more quantitative version, of course), then $\mu_{h}\left(S_{\alpha} \cap U\right)=\infty$ for all $\alpha$ and all open sets $U \subset(0, \infty)$. In fact, we even have that $S_{\alpha} \cap U$ is not $\sigma$-finite with respect to $\mu_{h}$.

I do not think that this extension of Theorem 4.2 gives much additional insight, but it does provide explicit examples where the set $S_{\alpha}$ is arbitrarily large in the measure theoretic sense (given the restriction that $\left|S_{\alpha}\right|=0$ ).

\section{REFERENCES}

[1] E. A. Coddington and N. Levinson, Theory of Ordinary Differential Equations, McGraw-Hill, New York, 1955. MR 16:1022b

[2] R. del Rio, S. Jitomirskaya, Y. Last, and B. Simon, Operators with singular continuous spectrum, IV. Hausdorff dimensions, rank one perturbations, and localization, J. d'Analyse Math. 69 (1996), 153-200. MR 97m:47002

[3] R. del Rio, B. Simon, and G. Stolz, Stability of spectral types for Sturm-Liouville operators, Math. Research Letters 1 (1994), 437-450. MR 95i:47084

[4] D. J. Gilbert and D. B. Pearson, On subordinacy and analysis of the spectrum of onedimensional Schrödinger operators, J. Math. Anal. Appl. 128 (1987), 30-56. MR 89a:34033

[5] A. Y. Gordon, S. A. Molchanov, and B. Tsagani, Spectral theory of one-dimensional Schrödinger operators with strongly fluctuating potentials, Funct. Anal. Appl. 25 (1992), 236-238. MR 93a:34097

[6] S. Y. Jitomirskaya and Y. Last, Dimensional Hausdorff properties of singular continuous spectra, Phys. Rev. Letters 76 (1996), 1765-1769. MR 96k:81041

[7] W. Kirsch, S. A. Molchanov, and L. Pastur, One-dimensional Schrödinger operators with high potential barriers. Operator Theory: Advances and Applications, Vol. 57, 163-170. Birkhäuser Verlag, Basel, 1992. MR 94f:34167

[8] A. Kiselev, Y. Last, and B. Simon, Modified Prüfer and EFGP transforms and the spectral analysis of one-dimensional Schrödinger operators, Comm. Math. Phys. 194 (1998), 1-45. CMP 98:14

[9] Y. Last, Quantum dynamics and decompositions of singular continuous spectra, J. Funct. Anal. 142 (1996), 406-445. MR 97k:51044

[10] Y. Last and B. Simon, Eigenfunctions, Transfer Matrices, and Absolutely Continuous Spectrum of One-Dimensional Schrödinger Operators, to appear in Inv. Math.

[11] S. Molchanov, One-dimensional Schrödinger operators with sparse potentials, preprint (1997).

[12] S. N. Naboko, Dense point spectra of Schrödinger and Dirac operators, Theor. and Math. Phys. 68 (1986), 646-653. MR 88h:81029 
[13] D. B. Pearson, Singular Continuous Measures in Scattering Theory, Comm. Math. Phys. 60 (1978), 13-36. MR 58:4076

[14] M. Reed and B. Simon, Methods of Modern Mathematical Physics, III. Scattering Theory, Academic Press, London-San Diego, 1979. MR 80m:81085

[15] C. Remling, Relationships between the $m$-function and subordinate solutions of second order differential operators, J. Math. Anal. Appl. 206 (1997), 352-363. MR 97m:34052

[16] C. Remling, A probabilistic approach to one-dimensional Schrödinger operators with sparse potentials, Comm. Math. Phys. 185 (1997), 313-323. MR 98j:3474

[17] C. Remling, Some Schrödinger operators with power-decaying potentials and pure point spectrum, Comm. Math. Phys. 186 (1997), 481-493. MR 98k:34140

[18] C. A. Rogers, Hausdorff Measures, Cambridge University Press, London, 1970. MR 43:7576

[19] W. Rudin, Real and Complex Analysis, McGraw-Hill, Singapore, 1987. MR 88k:00002

[20] B. Simon, Operators with singular continuous spectrum, VII. Examples with borderline time decay, Comm. Math. Phys. 176 (1996), 713-722. MR 97a:47006

[21] B. Simon, Some Schrödinger operators with dense pure point spectrum, Proc. Amer. Math. Soc. 125 (1997), 203-208. MR 97c:34179

[22] B. Simon and G. Stolz, Operators with singular continuous spectrum, V. Sparse potentials, Proc. Amer. Math. Soc. 124 (1996), 2073-2080. MR 97a:47004

[23] G. Stolz, Bounded solutions and absolute continuity of Sturm-Liouville operators, J. Math. Anal. Appl. 169 (1992), 210-228. MR 93f:34141

[24] W. F. Stout, Almost Sure Convergence, Academic Press, New York, 1974. MR 56:13334

[25] J. Weidmann, Spectral Theory of Ordinary Differential Operators, Lecture Notes in Math., Vol. 1258, Springer-Verlag, Berlin-Heidelberg, 1987. MR 89b:47070

[26] A. Zygmund, A remark on Fourier transforms, Proc. Cambridge Phil. Soc. 32 (1936), 321327.

Universität Osnabrück, Fachbereich Mathematik/Informatik, 49069 Osnabrück, GERMANY

E-mail address: cremling@mathematik.uni-osnabrueck.de 